\title{
Regional Agriculture Coupling Coordination Analysis Based on Sustainable Development: A Case Study of Sichuan, China
}

\author{
Fumin Deng ${ }^{1}$, Siyuan Jia ${ }^{1, \mathrm{a}}$, Qunxi Gong ${ }^{1,2, \mathrm{~b}^{*}}$ \\ ${ }^{1}$ Business School of Sichuan University, Chengdu 610065, Sichuan, China \\ ${ }^{2}$ Sichuan Haina Rendong Science and Technology Co. Ltd.
}

\begin{abstract}
As a basic industry for national development, the development of agriculture will reduce food insecurity, get rid of resource dependence and achieve broader development. This paper constructs a fourdimensional measurement system of food production, economic development, ecological protection, and social progress by considering food security. Comprehensive agricultural development in Sichuan Province from 2004 to 2018 was evaluated by using improved entropy method and coupling coordination degree model (CCD). The study found that: (1) From 2004 to 2018, comprehensive development of the four subsystems in Sichuan agriculture are different, ecological protection $(9.62 \%)>$ social progress $(3.34 \%)>$ economic development $(2.61 \%)>$ food production $(-0.44 \%)$; (2) The comprehensive development level of agriculture in Sichuan Province had been continuously improved from 2004 to 2018, with obvious phase characteristics; (3) The coordination development among subsystems advanced in volatility, which is adjusted from the medium to the optimal level of coordination. Finally, based on the above conclusions, Sichuan agricultural sustainable development can be improved from the perspective of food production, economic development and social progress on the basis of ecological protection.
\end{abstract}

\section{Introduction}

Agriculture is the foundation of national economic construction and development (h. Zhao, 2013). It is undeniable that with the development of modern technology, the application of chemical fertilizers, pesticides, machinery and so on can significantly increase food output(F. Li \& Yang, 2016), but they also cause imbalance in ecological, economic and social development. In order to ensure the coordinated development of regional agricultural system, it is necessary to determine the development strategy and policy by measuring the comprehensive agriculture development and exploring the evolution agricultural coordination tendency.

Scholars' research on agricultural development mainly focuses on the construction of agricultural indicator system and the selection of measurement methods. From the perspective of the regional agriculture indicator construction, the indicator system mainly focuses on three dimensions, namely economy, environment and society(M. Tang, 2019; Q. Tang \& He, 2019). On this basis, some studies have added agricultural subsidies(Q. Tang \& He, 2019), agricultural insurance (Huang, Yu, \& $\mathrm{Hu}$, 2019) and so on to evaluate the comprehensive development of agriculture. Diversification has become a common trend in the development of agricultural indicators. From the perspective of the research method in regional agriculture, (Liu, Qi, QiangFu, \& Li, 2019) assessed the resiliency of regional combinations of agricultural water resources and soil resources by using weighted Markov distance and grey relational models. (M. Li et al., 2020) established an uncertain multi-objective nonlinear optimization model to evaluate agricultural water and soil resources. By using the social metabolism theory, (Grace et al., 2020) showed that the recycling of compost would improve energy efficiency.

In general, scholars' researches on agricultural sustainable development are abundant in both indicator construction and research methods, but there are few studies on the coordinated development of indicators. In addition, coupling coordination within agriculture is rarely involves social subsystems, let alone the analysis of food production subsystem as the third perspective. Based on the deficiency of existing literature, the improved entropy weight method is used to objectively weight the indicators, and quantitative analyze the development of regional agriculture by using coupling coordination degree model (CCD) to conduct. The core of this paper is to integrate food production, economic development, ecological protection and social progress into agriculture system. Firstly, the comprehensive development of each subsystem was analyzed, and the Sichuan agriculture development was evaluated. Accordingly, this paper innovates from the perspective of research object and indicator system. (1) Construct the agriculture system form four dimentions: food production, economy development, ecology protection and society progress subsystems (2) The coupling coordination method is used to study the coordination relationship between subsystems

\footnotetext{
* Corresponding author: ${ }^{b}$ gongqunxi@stu.scu.edu.cn

2019225020033@stu.scu.edu.cn
} 


\section{Methodology}

\subsection{The indicator system of regional agriculture}

Based on the previous research of related journals, and considering the availability of data, this paper constructs an indicator system to analyze the development level and coupling relationship of each subsystem shown in Table 1.

Five indicators are selected to evaluate the food production from the perspective of food production scale and yield. For economic development, six indicators are selected from the perspective of economic growth, output ratio and infrastructure. To describe agricultural ecological protection five indicators were selected from ecological driving force, ecological state and ecological response. For the progress of agricultural society, five indicators are selected based on people's quality of life and agricultural social security.

Table1 Indicator and weight of agriculture system

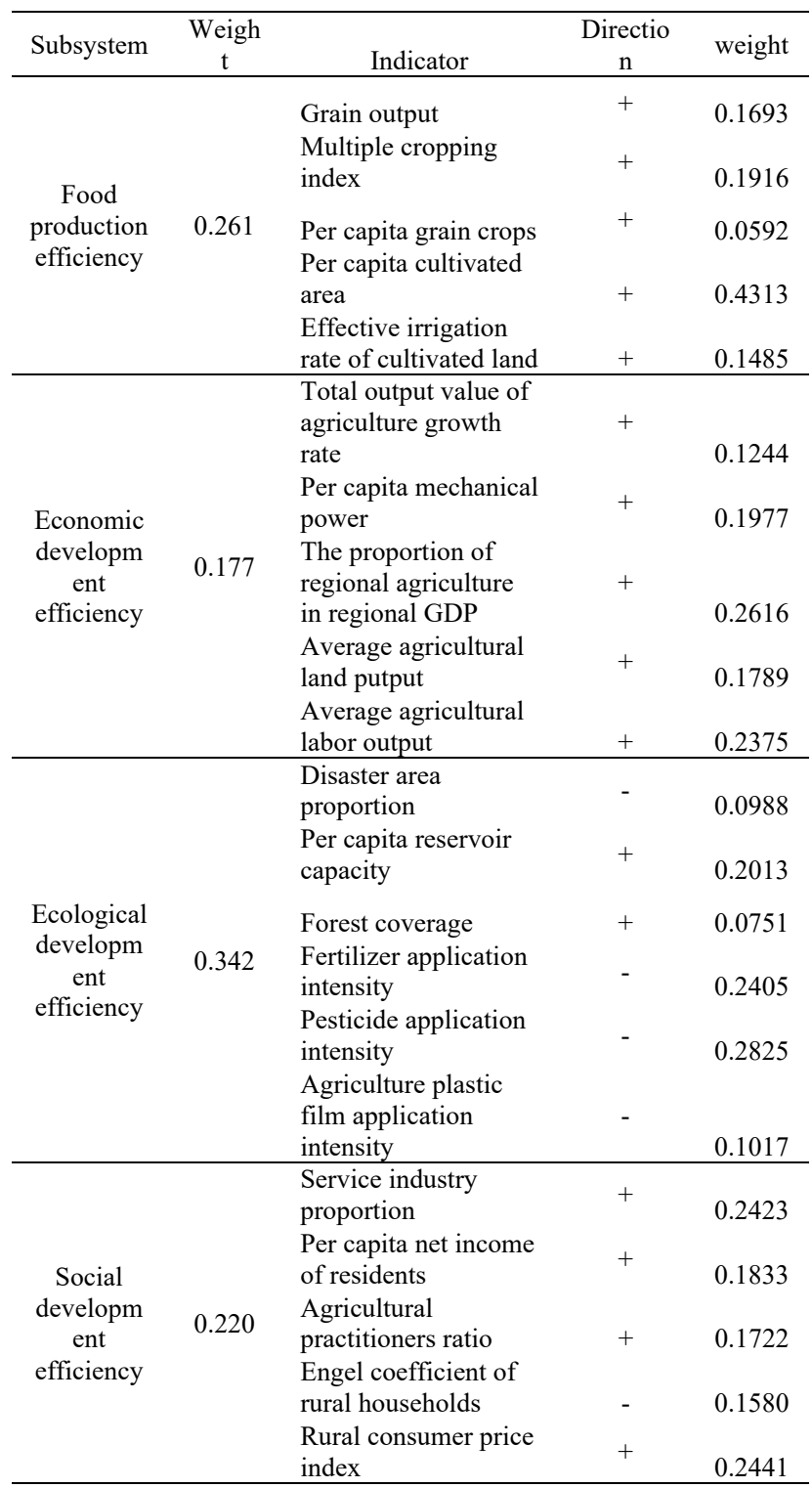

\subsection{Entropy weight method}

Entropy weight method can eliminate the influence of human factors on the indicator weights determination and make it with higher reliability. In this paper, the subsequent procedure is used the study of(Xing, Xue, \& $\mathrm{Hu}$, 2019).First, the initial matrix A formed with the original data is shown below.

$$
A=\left(X_{\mathrm{tij}}\right)_{n \times m}=\left(\begin{array}{ccc}
X_{111} & \cdots & X_{11 m} \\
\vdots & \ddots & \vdots \\
X_{n 41} & \cdots & X_{n 4 m}
\end{array}\right)
$$

Where $\mathrm{t}=1,2, \ldots, \mathrm{n} ; \mathrm{i}=1,2,3,4 ; \mathrm{j}=1,2, \ldots, \mathrm{m}, \mathrm{n}$ is the number of year and $\mathrm{m}$ is the number of indicators. $X_{\mathrm{tij}}$ represents the $j$-th indicator of the $i$-th subsystem in the year $t$.

Raw data was collected to carry out the standardized treatment shown as equation (2) and (3). At the same time, in order to meet the requirement of logarithm, the entropy method should be improved (Yuan \& Qi, 2013).

Positive index:

$$
X_{i j}^{\prime}=\frac{X_{i j}-\min \left(X_{1 i j}, X_{2 i j}, \cdots, X_{n i j}\right)}{\max \left(X_{1 i j}, X_{2 i j}, \cdots, X_{i j j}\right)-\min \left(X_{1 i j}, X_{2 i j}, \cdots, X_{t i j}\right)}+0.0
$$

Negative index:

$$
X_{i j}^{\prime}=\frac{\max \left(X_{1 i j}, X_{2 i j}, \cdots, X_{n i j}\right)-X_{i j}}{\max \left(X_{1 i j}, X_{2 i j}, \cdots, X_{n i j}\right)-\min \left(X_{1 i j}, X_{2 i j}, \cdots, X_{n i j}\right)}+0
$$

Where $X_{t i j}$ is the original data, $X_{t i j}^{\prime}$ is the processed data. Entropy weight method was used to obtain the competitiveness of each indicator and the subsystem weight by using equation (4) to (7).

$$
\begin{aligned}
& P_{t i j}=\frac{\mathrm{X}_{\mathrm{tij}}^{\prime}}{\sum_{\mathrm{t}=1}^{\mathrm{n}} \mathrm{X}_{\mathrm{tij}}^{\prime}} \\
& E_{i j}=-k \sum_{t=1}^{n}\left(P_{t i j} \ln P_{t i j}\right) \\
& w_{i j}=\frac{1-E_{i j}}{m-\sum_{j=1}^{m} E_{i j}} \\
& w_{i}=\sum_{j=1}^{m} w_{i j}
\end{aligned}
$$

Where $P_{t i j}$ is the proportion of $j$-th index parameter in $i$-th subsystem. entropy $E_{i j}$ calculated in equation (5), where $\mathrm{k}$ is constant, and $\mathrm{k}=\frac{1}{\operatorname{lnm}}, 0 \leq \mathrm{E}_{\mathrm{ij}} \leq 1$. The information weight $\mathrm{w}_{\mathrm{ij}}$ can be calculated by formula (6), and the weight of each subsystem can be calculated by formula (7).

\subsection{Coupling coordination degree model}

Coupling is a phenomenon that occurs when two or more systems interact over a period of time. The formula is as follows(Song, Zhou, Liu, Siehr, \& Qi, 2018): 


$$
\begin{aligned}
& \mathrm{C}=4 \times \sqrt[4]{\frac{u_{1} u_{2} u_{3} u_{4}}{\left(u_{1}+u_{2}+u_{3}+u_{4}\right)^{4}}} \\
& \mathrm{D}=\sqrt{C \times T}
\end{aligned}
$$

Where $\mathrm{C}_{\mathrm{i}}$ is the coupling degree(CD) among the subsystems, $u_{1}, u_{2} u_{3}, u_{4}$ is the comprehensive index of four subsystems. D represents CCD, T is the system comprehensive coordination index, $u_{i}$ is the subsystem comprehensive index. The calculation formula (10) (11) shown as follows.

$$
\begin{aligned}
& u_{k}=\sum_{j=1}^{m} w_{i j} X_{i j}^{\prime} \\
& \mathrm{T}=\alpha u_{1}+\beta u_{2}+\gamma u_{3}+\delta u_{4}
\end{aligned}
$$

Where, $\mathrm{k}$ is the number of systems, $\mathrm{k}=1,2,3,4 . \mathrm{w}_{\mathrm{ij}}$ is the weight of the corresponding index within the k-th subsystem. $\alpha, \beta, \gamma$ and $\delta$ represents the weight of food, economy, ecology and social subsystem respectively. In this study, it is considered that each subsystem plays an equally important role in the agricultural resources, so $\alpha=\beta=\gamma=\delta=0.25$. Median segmentation method (Ma, Jin, \& Liu, 2012) was used to classify the coupling coordination types of agricultural subsystems.

Table 2 Discriminating standards of coupling coordination degree

\begin{tabular}{cccc}
\hline $\begin{array}{c}\text { Coupling } \\
\text { degree } \\
(\mathrm{C})\end{array}$ & Coupling type & $\begin{array}{c}\text { Coupling } \\
\text { coordination } \\
\text { degree (D) }\end{array}$ & $\begin{array}{c}\text { Type of coupling } \\
\text { coordination }\end{array}$ \\
\hline $0<\mathrm{C} \leq 0.3$ & $\begin{array}{c}\text { Lower level } \\
\text { coupling }\end{array}$ & $0<\mathrm{D} \leq 0.45$ & $\begin{array}{c}\text { Lower coordination } \\
\text { coupling }\end{array}$ \\
$0.3<\mathrm{C} \leq$ & $\begin{array}{c}\text { Medium } \\
\text { coupling level }\end{array}$ & $0.45<\mathrm{D} \leq 0.65$ & $\begin{array}{c}\text { Medium coordination } \\
\text { coupling }\end{array}$ \\
0.5 & Higher & $0.65<\mathrm{D} \leq 0.80$ & $\begin{array}{c}\text { Higher coordination } \\
\text { coupling }\end{array}$ \\
$0.5<\mathrm{C} \leq$ & $\begin{array}{c}\text { coupling level } \\
\text { Benign level } \\
\text { coupling }\end{array}$ & $0.80<\mathrm{D} \leq 1$ & $\begin{array}{c}\text { Optimal coordination } \\
\text { coupling }\end{array}$ \\
$0.8<\mathrm{C} \leq 1$ & & &
\end{tabular}

\subsection{Study area and data source}

\subsubsection{Study area}

Sichuan, with mountains as the main landform, has a great vertical change in climate, forming a variety of agricultural industry types. According to the 2019 China Rural Statistics Yearbook, The total agricultural output value of Sichuan reached 719.56 billion yuan in 2018, is the third-highest output in China, among which the output of pork ranked first, making it an important major grain producing area in China. In recent years, the unbalanced utilization of agricultural resources has restricted the development of Sichuan. Compared with other provinces in China, crop diseases and insect pests occur frequently in Sichuan due to climate, leading to excessive dependence of chemical inputs such as fertilizers, pesticides and plastic film by farmers, resulting in an increased load of agricultural ecological resources and environment(Y. Zhao \& Lv, 2019). In addition, with the acceleration of urbanization, the variety of permanent rural population showed an obvious downward trend. In view of this, the relation between food, economy, zoology, society is getting attention increasingly. The research results based on this province can also provide reference for the sustainable management of agricultural resources in other major grain-producing areas of the world.

\subsubsection{Data source}

The time series of this study is from 2004 to 2019. The original data required in this paper are all from China Rural Statistical Yearbook, China Statistical Yearbook, China Environmental Statistical Yearbook and Sichuan Statistical Yearbook over those years.

\section{Results}

According to formulas (1) to (7), the weights of each system index in Sichuan province are calculated respectively, as shown in Table 1. After establishing the evaluation index, the comprehensive evaluation value of food production $\left(u_{1}\right)$, economic development $\left(u_{2}\right)$, ecological protection $\left(u_{3}\right)$, social progress $\left(u_{4}\right)$, CD (C) and CCD (D) from 2004 to 2018 were calculated respectively according to formula (8) to (11). the result, shown in Figure 1 and Figure 2.

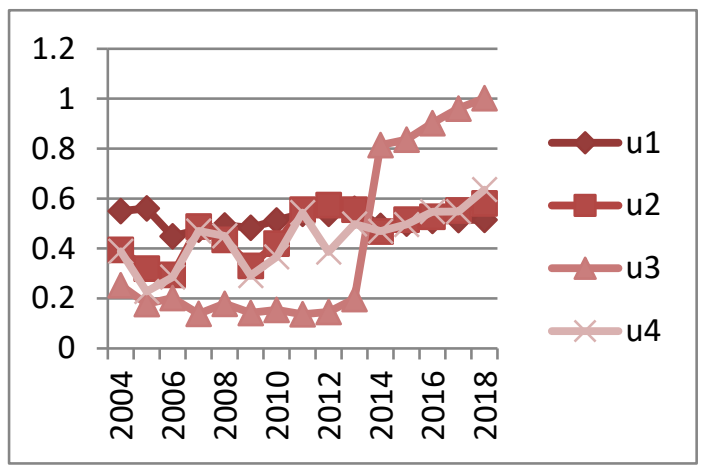

Figure 1 Comprehensive development trend of 4 subsystem

From the perspective of subsystem growth rate, the annual average growth rate of comprehensive evaluation on food production is $-0.44 \%$, economic development $2.61 \%$, ecological protection $9.62 \%$, and social progress $3.34 \%$. From the perspective of growth trend, economic development and social progress showed a fluctuating upward trend, while ecological development showed a sudden increase trend. The development of grain production showed a slow negative growth trend.

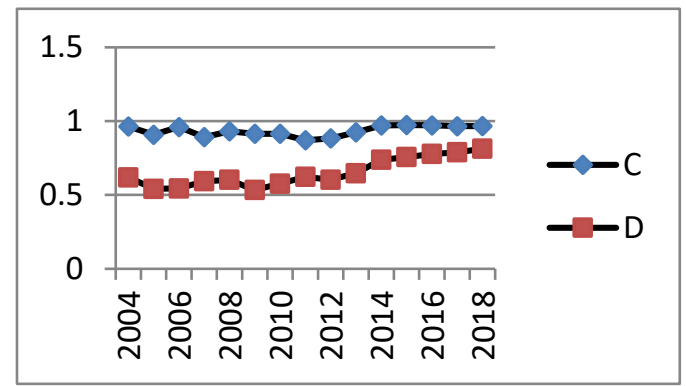

Figure 2 Agricultural CD and CCD in Sichuan from 2004 to 2018 
The coupling relationship between agricultural food production, economic development, ecological protection and social progress in Sichuan Province is shown in Figure 2. The CD of four subsystem increased from 0.964 to 0.967 with an average growth rate of 0.0002 . From 2004 to 2018, the minimum coupling of the four subsystems was 0.871 , the highest coupling was 0.974 , and the CD was in the benign level coupling $(0.8<\mathrm{C} \leqslant 1)$, which indicated that the agricultural systems in Sichuan are closely related and have a great influence on each other. The coupling coordination degree increased from 0.618 to 0.813 , and the average growth rate was 0.0184 . This shows that the CCD between the systems has increased from medium coordination coupling $(0.45<\mathrm{D} \leqslant 0.65)$ to optimal coordination coupling $(0.80<\mathrm{D} \leqslant 1)$. The development of each subsystem has a positive impact on each other. Their development differences are gradually narrowing, and their development status and speed are tending to be the same.

\section{Discussion}

\subsection{Subsystem comprehensive evaluation analysis}

Based on the main influencing factors of each system according to the weight, this paper will interpret the development of food production subsystem, economic development subsystem, ecological protection subsystem and social progress subsystem from two stages.

In the first stage (2004-2013), for the food production subsystem, although the increase of cultivated land area and sown area increased the food yield, the decrease of multiple cropping index reduced the utilization rate of cultivated land, which eventually led to the slow development of food production subsystem. From the perspective of the subsystem of agricultural economy and social progress, factors such as the increase of average agricultural labor output, labor transfer and decrease of agricultural population make economic and social development showed fluctuating growth. For the agricultural ecological protection subsystem, the use of pesticides, chemical fertilizers and plastic film increased year by year, resulting in a fluctuating downward trend. Although, the increase of these factors stimulated the rapid growth of food production in Sichuan, caused environmental pollution. In addition, the international financial crisis in 2008 and the Wenchuan earthquake with $\mathrm{M}=8.0$ have had an impact on the agricultural economy, society, ecology and food development in Sichuan. As can be seen from Figure 1, social and economic subsystems did not gradually improve until 2010. And with the passage of time, the development of Sichuan's agriculture was supported by the development of food production transformed into the development of agricultural economy as the driving force in 2013.

In the second stage (2014-2018), the subsystem of food production, economic development, ecological protection, and social progress all show an upward trend, but there are obvious differences in the extent of the rise.
In contrast, the ecological development showed a stronger upward trend from the bottom, and the other three subsystems basically kept synchronous tendency. The driving force of Sichuan agriculture has gradually changed from economic to ecological development. In 2013, the outbreak of " $\mathrm{h} 7 \mathrm{n} 9$ " avian influenza virus and the earthquake of magnitude 7 in Sichuan Province had a certain impact on the economic and social recovery and food harvest in the second year. But China proposed "one belt, one road" construction strategy in the same year. The external demand of environmental protection has promoted the industrial upgrading in the central and western regions, it can be seen from Figure 1 that the ecological environment and the ecological benefits have been significantly improved. In addition, in 2016, China's "the 13th five-year plan" formally put forward the goal of "reducing fertilizer and medicine, increasing quality and efficiency", and incorporated food production and environmental protection into the specific planning. However, compared with the other three subsystems, due to the excessive use of pesticides and fertilizers, as well as the influence of the law of diminishing marginal benefits, the quality of cultivated land is continuously affected, and the development of food production subsystem is the slowest. In general, food production, economy development and society progress have achieved stable growth under the driving effect of ecological protection.

\subsection{Coupling degree and coupling coordination analysis}

The CD and CCD of the four subsystems can be analyzed from its fluctuation and growth. From 2004 to 2018, the fluctuation trend of CD and CCD gradually slowed down and got closer to 1 . There is a high degree of positive interaction among the four subsystems, and this interaction is more and more stable. The growth of CCD level increases obviously, from moderate coordination degree to optimal coordination coupling level. In addition, the growth trend changed from a fluctuating rise from 2004 to 2013 to a stable rise from 2014 to 2018 . After the continuous interaction of subsystems in Sichuan, the agriculture development is becoming more and more coordinated.Although the four subsystems show a strong interaction relationship, except the ecological protection subsystem, the comprehensive development of the other three subsystems did not reach 0.7. Therefore, the agriculture development did not reach the optimal coordination level until 2018. In a word, Sichuan agricultural system is at the low level in optimal coordination coupling range, and there is still a great space to improvement.

\section{Conclusion, policy suggestion and deficiency}

\subsection{Conclusion}

To realize sustainable agricultural development, this paper makes an empirical analysis of the coupled and 
coordinated development of food production, economic development, ecological protection and social progress in Sichuan province from 2004 to 2018 by using the improved entropy method and the coupling coordination model, so as to reveal the coordinated situation in Sichuan Province agricultural. The research conclusions are as follows:

(1) From the perspective of comprehensive development of subsystems, the development rate is different, among which ecological protection $(9.62 \%)>$ social progress $(3.34 \%)>$ economic development $(2.61 \%)>$ food production $(-0.44 \%)$. Each subsystem increased with fluctuation from 2004 to 2013, and it achieved a steady increase from 2014 to 2018.

(2) From the coupling relationship between subsystems, agriculture in Sichuan has always been in a highly coupled state. From 2004 to 2018, agricultural development shifted from being driven by food production to economic development, and finally achieved stable growth under the driving role of ecological protection.

(3) From the perspective of CCD, the four subsystems of agricultural development in Sichuan has shifted from Medium coordination coupling to optimal coordination coupling, among which the growth was fluctuant from 2004 to 2013 and stable from 2014 to 2018. The sustainable development of agriculture in Sichuan can be improved from the contradiction between environment, labor transfer and grain yield.

\subsection{Deficiency}

Aiming at the food security in agriculture, this paper constructs a comprehensive evaluation of regional agriculture including food production, economic development, ecological protection and social progress. In the future, it will also study and improve regional agricultural development from other aspects of agriculture such as animal husbandry.

\section{References}

1. Grace, B. V., Andrew, D. K., David, L. G., Mario, E. d. H.-M., Audrey, M. M., Maribel, A., \& Lilian, A. (2020). Landscape social-metabolism in food-energywater systems: Agricultural transformation of the Upper Snake River Basin. Science of The Total Environment, 705(25), e135817.

2. Huang, J., Yu, G., \& Hu, D. (2019). The integrated and coordinated development of agricultural modernization and agricultural insurance: A case study of Xinjiang. Research of Agricultural Modernization, 40(02), 197-205.

3. Li, F., \& Yang, X. (2016). Analysis of the Partial Least-Square Regression (PLS) on Influencing Factors of Grain Output in Guizhou Province. Journal of Sichuan Normal University(Natural Science), 39(1), 151-158.

4. Li, M., Fu, Q., Singh, V. P., Liu, D., Li, T., \& Zhou, Y. (2020). Managing agricultural water and land resources with tradeoff between economic, environmental, and social considerations: A multiobjective non-linear optimization model under uncertainty. Agricultural Systems, 178, e102685.

5. Liu, D., Qi, X., QiangFu, \& Li, M. (2019). A resilience evaluation method for a combined regional agricultural water and soil resource system based on Weighted Mahalanobis distance and a Gray-TOPSIS model. Journal of Cleaner Production, 229, 667-679.

6. Ma, L., Jin, F. J., \& Liu, Y. (2012). Spatial pattern and industrial sector structure analysis on the coupling and coordinating degree of regional economic development and environment pollution in China. Acta Geographica Sinica, 67(10), 1299-1307.

7. Song, Q., Zhou, N., Liu, T., Siehr, S. A., \& Qi, Y. (2018). nvestigation of a "coupling model" of coordination between low-carbon development and urbanization in China. Energy Policy, 121(346-354).

8. Tang, M. (2019). Research on Coupling Coordination of Shanxi Agricultural Ecology-Economy-Society System. Shanxi University of Science and Technology,

9. Tang, Q., \& He, P. (2019). Analysis of Coupling Coordination Development among the Agricultural Environment Grain Production-Agricultural Subsidy: A Case Study of Hubei Province. Ecological Economy, 35(9), 116-120.

10. Xing, L., Xue, M., \& Hu, M. (2019). Dynamic simulation and assessment of the coupling coordination degree of the economy-resourceenvironment system: Case of Wuhan City in China. J Environ Manage, 230, 474-487.

11. Yuan, J. H., \& Qi, C. J. (2013). Dynamic Assessment of Regional Agricultural Sustainability of Hunan Province Based on Entropy Method. Resources and Environment in the Yangtze Basin, 2013(22), 152157.

12. Zhao, h. (2013). The Study of the Coordinative Development of Anhui's Urbanization and Agriculture Modernization. Anhui University,

13. Zhao, Y., \& Lv, H. (2019). Evaluation on Comparative Advantage of Grain Production in Sichuan Province and Its Main Influencing Factors. Agricultural Economics and Management(05), 64 -73. 